\title{
Gamma-ray burst spectral evolution in the internal shock model: confrontation with Fermi observations
}

\section{Željka Bošnjak*}

AIM (UMR 7158 CEA/DSM-CNRS-Université Paris Diderot) Irfu/Service d'Astrophysique,

Saclay 91191 Gif-sur-Yvette Cedex, France

E-mail: zeljka.bosnjakecea.fr

\section{Frédéric Daigne}

Institut d'Astrophysique de Paris, UMR 7095 Université Pierre et Marie Curie-Paris 6 - CNRS, 98 bis boulevard Arago, 75014 Paris, France

E-mail: daignediap.fr

\begin{abstract}
Using a time-dependent numerical model where the prompt gamma-ray burst emission is calculated in the framework of the internal shock model on a broad energy range (from soft X-ray to $\mathrm{GeV}$ energies), we compute gamma ray burst lightcurves and time-evolving spectra. We show how the spectral evolution in this model is determined by the evolution of the physical conditions in the shocked regions and by the dominant radiative process for the effective microphysics parameters. Model predictions are confronted to the observations in the standard sub-MeV energy range, as well as with high energy bands observed by Fermi. In the soft gamma-ray range we considered the evolution of the pulse shape in different energy channels and the empirical hardness-intensity correlation; the scenario where the soft gamma-ray component is due to synchrotron radiation from shock accelerated electrons gives the best agreement with observations. In this scenario a variable inverse Compton component is expected at high energies (>100 MeV). We examine the effect of this component on the observed light curve and spectral properties. In particular, we investigate if the properties of Fermi LAT observations (the delayed onset of high energy component, its prolonged duration with respect to GBM emission) can be accomodated within our model.
\end{abstract}

25th Texas Symposium on Relativistic Astrophysics - TEXAS 2010

December 06-10, 2010

Heidelberg, Germany

\footnotetext{
* Speaker.
} 


\section{Introduction}

In about two years of operation, the Fermi gamma-ray space telescope observations of gammaray bursts (GRBs) have already provided significant insights in the high-energy $(>100 \mathrm{MeV})$ behavior. There are several new important spectral and temporal properties [1] revealed by the observations of the Fermi Large Area Telescope (LAT) [2] in the high energy band $(20 \mathrm{MeV}-300 \mathrm{GeV})$ : (i) the onset of the LAT emission is often shifted to later times with respect to the prompt emission in the sub-MeV range detected by Fermi Gamma-ray Burst Monitor (GBM); (ii) a time resolved spectral analysis showed that no additional spectral component in LAT energy band is required in most of the events (for an exception see e.g. [3] for GRB 090902B). The spectral shape is consistent with the extension of a high energy power law of empirical Band function [ $[$ ] to LAT energies; (iii) the $>100 \mathrm{MeV}$ emission lasts long after the sub-MeV prompt emission (for an exception see e.g. [1] for GRB 090217). Following these discoveries several new mechanisms for high energy photons production in GRBs were proposed.

Theoretical models predict that prompt gamma-ray burst emission is produced in the expansion of the ultrarelativistic $(\Gamma \geq 100)$ unsteady outflow. The fundamental open questions concern the composition of the ejecta (baryonic matter or a Poynting flux) and the energy dissipation mechanism (the emission from internal shocks, the up-scattered photospheric emission or the magnetic dissipation). For the high energy emission there are two main classes of models involving leptonic or hadronic origin. In leptonic models the high energy spectral component can be produced e.g. by synchrotron self-Compton emission where the seed photons for inverse Compton scattering originate from different sources (e.g. [5], [6]) or by synchrotron process in the external forward shock (e.g. [7], [8], [9]). Models with the hadronic origin of the high energy emission involve processes related to high-energy protons (e.g. [10], [11]).

In this work we examine whether the light curves and spectra derived in the framework of the internal shock model [12] for GRBs can explain the observed spectral and temporal properties of gamma-ray bursts on a broad energy range as observed by Fermi satellite. In the internal shock model the initial inhomogenities in the distribution of the Lorentz factor within the relativistic outflow lead to creation of shock waves; a fraction of the kinetic energy which is dissipated in the shock waves is radiated and produces the prompt GRB. We focus on the most discussed version of the internal shock model where the radiation is due to shock-accelerated electrons in optically thin conditions. We use a numerical model [13] that was developed for the derivation of synthetic gamma-ray burst spectra and light curves by combining a detailed calculation of the dynamical evolution of the relativistic flow with the time-dependent radiative code including most relevant processes (adiabatic cooling, synchrotron radiation and self-absorption, inverse Compton scatterings and $\gamma \gamma$ annihilation). In our model the instantaneous photon spectrum is determined by the actual physical conditions in the emitting region, and it evolves in time as the physical conditions within the wind evolve with its expansion. The observed time-integrated spectrum includes the contributions from all photon spectra emerging from the relativistic outflow.

In [13], we explored the large parameter space of internal shocks and identified the class of models that can potentially accomodate different features of high energy spetra observed by Fermi LAT detector. This class of models is characterized by a dominant sub-MeV spectral component due to synchrotron emission at $\sim$ a few $100 \mathrm{keV}$ and a high energy $>100 \mathrm{MeV}$ spectral compo- 
nent due to inverse Compton scatterings. The relative luminosity of the synchrotron and inverse Compton components varies over the burst duration, since the parameters determining the physical conditions (and therefore the efficiency of various processes) in the shocked medium evolve. Here we examine how the synchrotron spectrum is modified in the presence of the inverse Compton scatterings and what are the resulting spectral parameters in the observer frame. One of the important consequences is the steepening of the low energy power law describing the spectra below the peak. As demonstrated in [14], the steep spectral slopes are reached when electrons suffer inverse Compton losses in the Klein-Nishina regime; it requires that a large fraction of the shock dissipated energy $(\sim 10-30 \%)$ is attributed to a small fraction of electrons $\sim 1 \%)$ and that the magnetic field energy density is very low $(<0.1 \%)$. We examine the effect of this additional radiative component in the light curves corresponding to the Fermi spectral bands.

The synthetic models can be tested against the empirical correlations among the observed GRB properties, e.g. the correlation between the pulse width and energy ([15]; [16]), the hardenssfluence correlation ([17]; [18]), and the hardness-intensity correlation ([19]; [18]). The possibility of our numerical model to produce synthetic light curves in different energy bands allows us to examine if the internal shock model could account for the properties observed in very high energy bands $(>100 \mathrm{MeV}$ ) by Fermi LAT detector (e.g. delayed onset of the emission and prolonged pulse duration with respect to sub-MeV bands).

\section{Synthetic spectra and lightcurves}

Using our numerical model we produce synthetic light curves corresponding to Fermi GBM and LAT energy bands ( $8 \mathrm{keV}-260 \mathrm{keV} ; 260 \mathrm{keV}-5 \mathrm{MeV} ; 100 \mathrm{MeV}-200 \mathrm{GeV} ; 1-200 \mathrm{GeV}$ ) and spectra in the observer frame; we integrate subsequent instantaneous spectra on a short timescale $(\Delta t=0.25 \mathrm{~s}$ ), and as well on the overal pulse duration. In the following we are going to examine two examples of the single pulse burst, that are both characterized by a spectral peak at $\sim$ a few 100 $\mathrm{keV}$ due to synchrotron emission. A simple single pulse burst can be considered as a constituent of the more complex event. The burst is generated by a relativistic ejection lasting for $t_{w}=2 \mathrm{~s}$ with a constant $\dot{E}=5 \times 10^{53} \mathrm{erg} / \mathrm{s}$ and a Lorentz factor increasing gradually from 110 to 400 , starting with the wind ejection (see [13] for the details). We assume that in every collision (i) a fraction $\varepsilon_{e}=1 / 3$ of the dissipated energy is injected in a fraction $\zeta$ of the electrons that are accelerated to relativistic energies with a power law distribution of slope - $p$; (ii) a fraction $\varepsilon_{B}$ of the dissipated energy is used for magnetic field amplification. The assumed redshift in both models is $z=1$. We consider the following examples:

- Model I: $\zeta=8 \times 10^{-4}, \varepsilon_{B}=5 \times 10^{-4}, p=2.5$. The peak energy of the synchrotron component is $\simeq 400 \mathrm{keV}$;

- Model II: we examine the effect of varying microphysics parameters (see [20]) and assume that the fraction of the accelerated electrons is varying according to the energy $\varepsilon^{*}$ dissipated in a shock, $\zeta=\zeta_{0} \times\left(\varepsilon^{*} / 100 \mathrm{MeV}\right.$ per proton $)$. We assume $\zeta_{0}=9 \times 10^{-4}, \varepsilon_{B}=10^{-5}, p=$ 3.5. The synchrotron component peak energy is $\simeq 200 \mathrm{keV}$. 

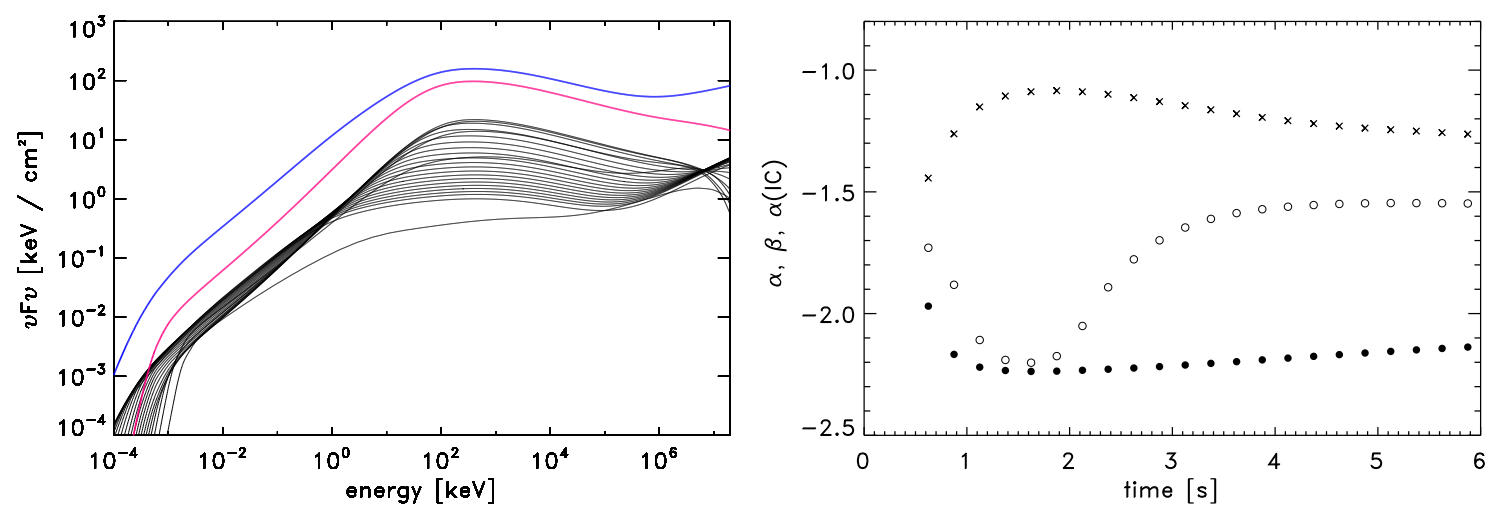

Figure 1: Left: Time-integrated and time-resolved spectra for Model I. Blue line corresponds to spectral integration on the interval $(0.5$ - 6) s (i.e. over whole duration of the emission), and red line corresponds to spectral integration on $(1-2.5) \mathrm{s}$ (i. e. around the pulse maximum in the Fermi GBM band). Black lines correspond to spectra on short time integration (see text) covering the pulse duration. Right: Slopes of the time-resolved photon spectra: $\alpha$ (crosses) and $\beta$ (filled circels) are the slopes of the low- and high- energy power laws in Band representation [\], while $\alpha$ (IC) (empty circles) is the low energy spectral slope of the inverse Compton component occurring at high energies. It is possible to measure $\alpha(\mathrm{IC})$ at the time intervals in which inverse Compton component is prominent in the total spectrum.

\section{Results}

We show the observed synthetic spectra and the corresponding spectral parameters for Model I in Figure 1: as described by [14], the low energy spectral slope $\alpha$ approaches the value -1 in the presence of inverse Compton scatterings occuring in the Klein-Nishina regime. For the given intial distribution of the Lorentz factors within the wind, the magnetic field and the minimum Lorentz factor of the electron distribution are decreasing strongly with the dynamical evolution (see [13]), and the peak of the synchrotron component is expected to decrease over the pulse duration. However, due to the inverse Compton scatterings of the photons just below the synchrotron spectral peak, the peak energy remains approximately constant in this example. In Model II (Figure 2) we tested the assumption on the varying microphysics parameter $\zeta$, and the steeper power law distribution of relativistic electrons, $p=3.5$. For a simple synchrotron cooling spectrum the expected high energy photon index is $\beta=-(p+2) / 2=-2.75$; however in the presence of the underlying inverse Compton component, the high energy spectral power law component becomes less steep (Figure 2, right panel) and it is approaching $\beta \simeq-2.3$ (Bosnjak et al. in prep).

Figure 3 shows the light curves for Model II. With our numerical model it is possible to test the correlation between the pulse width and energy [16]. In the example shown on Figure 3, left panel, the pulse width at half maximum varies with the energy as $W(E) \propto E^{-0.2}$. The maximum of the light curve at high energy bands (as observed by Fermi LAT) can be shifted to later times with respect to the sub-MeV bands due to the presence of the inverse Compton component (Figure 3, right panel). Finally, in Figure 4 we show hardness-intensity correlation (HIC) for Model II pulse. Our numerical model allows to distinguish between the rise and the decay phases of the pulse, and also to follow the high latitude emission. The fit of the power law HIC for the decay of the pulse gives the value consistent with the results by [18]; it is important to notice here that 

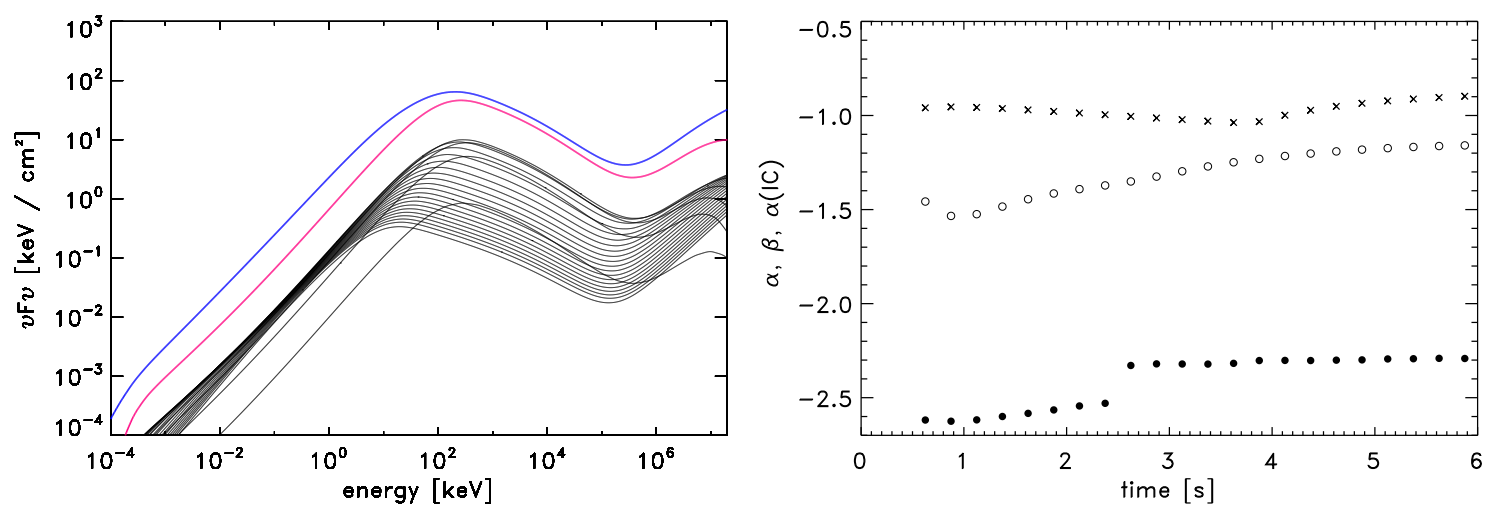

Figure 2: Left: Time-integrated and time-resolved spectra for Model II. Blue line corrseponds to spectral integration on the interval $(0.5$ - 6) s (i.e. over whole duration of the emission), and red line corresponds to spectral integration on $(0.5-2)$ s (i. e. around the pulse maximum in the Fermi GBM band). Black lines correspond to spectra on short time integration (see text) covering the pulse duration. Right: Slopes of the time-resolved photon spectra; the symbols have the same meaning as in Figure 1.

the slopes for the HIC differ depending on whether spectral/temporal properties are determined by the hydrodynamics of the flow or by the geometry of the emitting shells. The general agreement of the GRB properties derived in the internal shock model with the observations encourages its application to the individual gamma-ray bursts observed by Fermi.

\section{Acknowledgments}

The authors acknowledge the French Space Agency (CNES) for financial support.

\section{References}

[1] B.-B. Zhang et al., A Comprehensive analysis of Fermi gamma-ray burst data. I. Spectral components and their possible physical origins of LAT/GBM GRBs, [arXiv:1009.3338]

[2] W.-B. Atwood et al., The large area telescope on the Fermi gamma-ray space telescope emission, ApJ 697 (2009) 1071

[3] A. A. Abdo et al., Fermi observations of GRB 090902B: a distinct spectral component in the prompt and delayed emisson, ApJ 706 (2009) L138

[4] D. Band et al., BATSE observations of gamma-ray burst spectra. I. Spectral diversity, ApJ 413 (1993) 281

[5] Y.C. Zou et al., The possible high-energy emission from GRB 080319B and origins of the GeV emission of GRBs 080514B, 080916C and 081024B, MNRAS 396 (2009) 1163

[6] D. Guetta \& J. Granot, High-energy emission from the prompt gamma-ray burst, ApJ 585 (2003) 885

[7] P. Kumar \& R. Barniol Duran, On the generation of high-energy photons detected by the Fermi Satellite from gamma-ray bursts, MNRAS 400 (2009) L75

[8] G. Ghisellini et al., GeV emission from gamma-ray bursts: a radiative fireball?, MNRAS $\mathbf{4 0 3}$ (2010) 926 

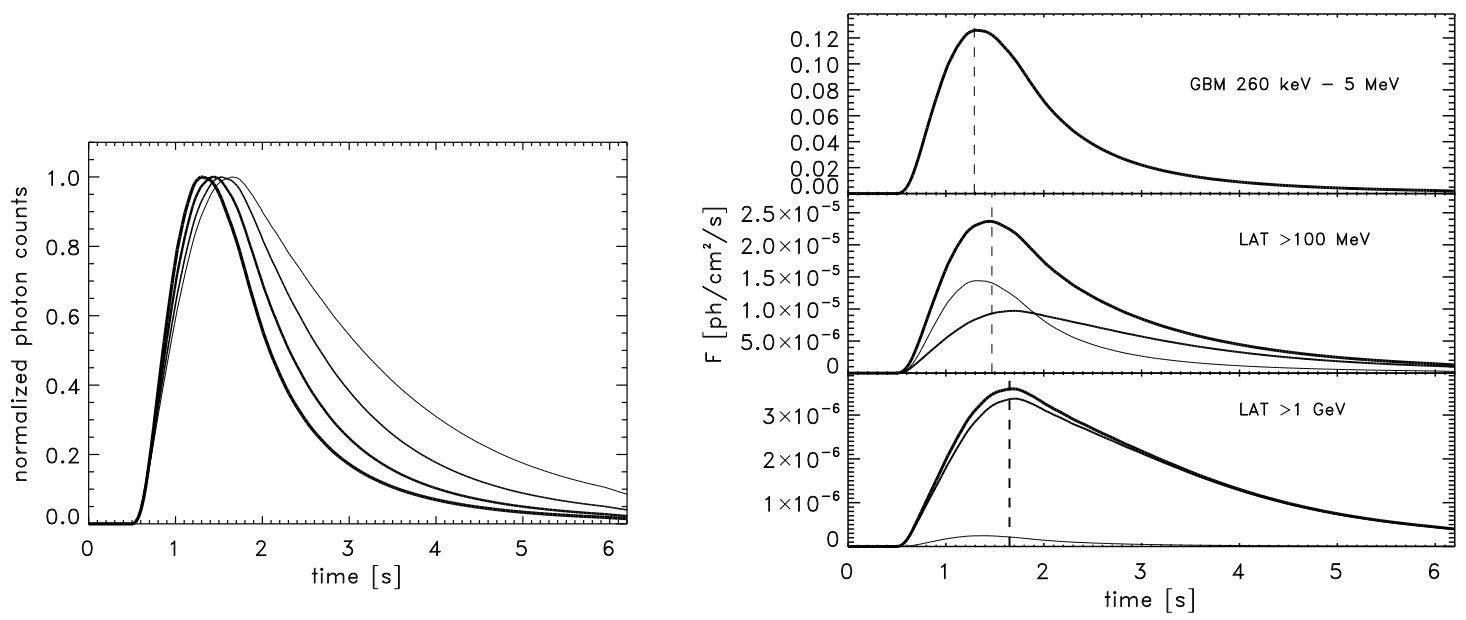

Figure 3: Model II. Left: Light curves corresponding to the four BATSE energy channels (20-50 keV; 50-100 keV; 100-300 keV; 300-2000 keV). The pulse shape in the highest energy band is shown with the thickest line, and the pulses in the lower energy bands by thiner lines. Right: Light curves corresponding to the Fermi GBM and LAT energy bands. The thickest line corresponds to the sum of the synchrotron and inverse Compton components; the thin line corresponds to the synchrotron component, while the mediumthick line represents the inverse Compton component. The dashed lines show the time at which light curve maximum occurrs. In this example the peak is shifted to later times in LAT energy bands, and the shift is for about $\sim 20 \%$ of the pulse duration $(\sim 0.2 \mathrm{~s})$.

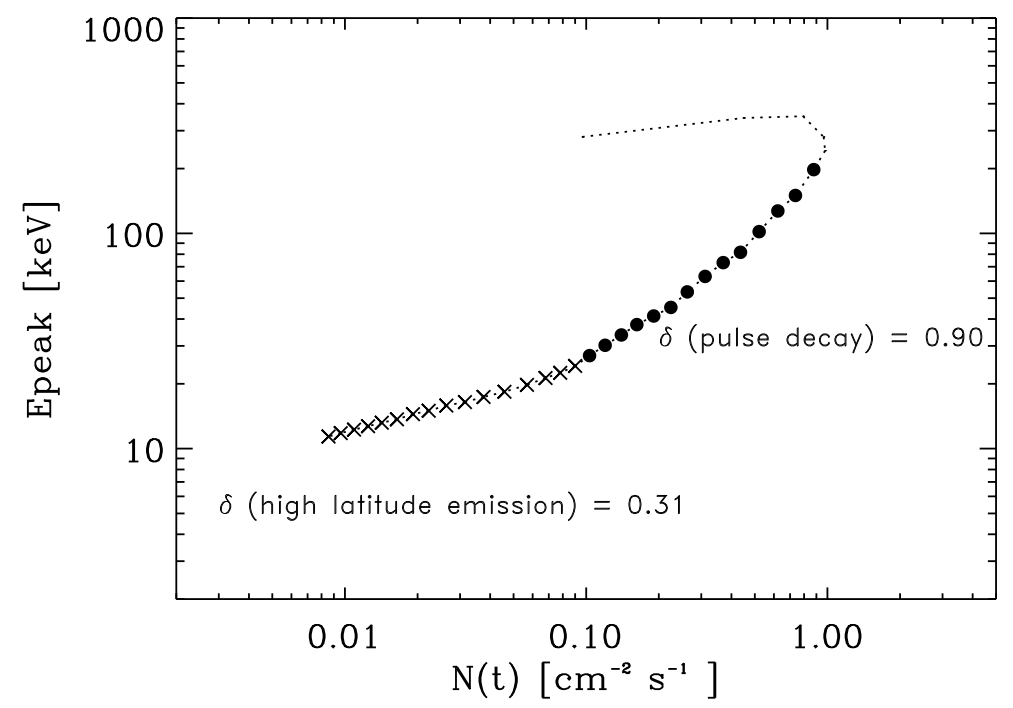

Figure 4: Hardness-intensity correlation for the Model II. We fitted a power law $E \propto N(t)^{\delta}$, where $\mathrm{E}$ is the peak energy and $\mathrm{N}(\mathrm{t})$ is the photon flux at time $t$ (see [18]), corresponding to the decay of the pulse and the high latitude emission respectively. 
[9] X.-Y. Wang, et al., GRB 080916C: On the radiation origin of the prompt emission from keV/MeV to GeV, ApJ 698 (2009) L98

[10] K. Asano et al., Hadronic models for the extra spectral component in the short GRB 090510, ApjL 705 (2009) L191

[11] S. Razzaque et al., GeV and higher energy photon interactions in gamma-ray burst fireballs and surroundings, ApJ 613 (2004) 1072

[12] P. Mészáros \& M.J. Rees, Unsteady outflow models for cosmological gamma-ray bursts, ApJ 430 (1994) L93

[13] Z. Bosnjak et al., Prompt high-energy emission from gamma-ray bursts in the internal shock model, A\&A 498 (2009) 677

[14] F. Daigne et al., Reconciling observed gamma-ray burst prompt spectra with synchrotron radiation?, A\&A 526 (2011) A110

[15] E.E. Fenimore et al., Expanding relativistic shells and gamma-ray burst temporal structure, ApJ 473 (1996) 998

[16] J. Norris et al., Attributes of pulses in long gamma-ray bursts, ApJ 459 (1996) 393

[17] E. Liang \& V. Kargatis, Dependence of the spectral evolution of $\gamma$-ray bursts on their photon fluence, Nat 381 (1996) 49

[18] F. Ryde \& R. Svensson, On the variety of the spectral and temporal behavior of long gamma-ray burst pulses ApJ 566 (2002) 210

[19] V. Kargatis, et al., Spectral evolution of GRB pulses, Ap\&SS 231 (1995) 177

[20] F. Daigne \& R. Mochkovitch, The physics of pulses in gamma-ray bursts: emission processes, temporal profiles and time-lags, MNRAS 296 (1998) 275 